\title{
Modulational instability and solitons in nonlocal media with competing nonlinearities
}

Esbensen, B. K.; Wlotzka, A.; Bache, Morten; Bang, Ole; Krolikowski, W.

Published in:

Advanced Photonics Congress

Publication date:

2012

Document Version

Publisher's PDF, also known as Version of record

Link back to DTU Orbit

Citation (APA):

Esbensen, B. K., Wlotzka, A., Bache, M., Bang, O., \& Krolikowski, W. (2012). Modulational instability and solitons in nonlocal media with competing nonlinearities. In Advanced Photonics Congress (pp. NW3D.4). Optical Society of America.

\section{General rights}

Copyright and moral rights for the publications made accessible in the public portal are retained by the authors and/or other copyright owners and it is a condition of accessing publications that users recognise and abide by the legal requirements associated with these rights.

- Users may download and print one copy of any publication from the public portal for the purpose of private study or research.

- You may not further distribute the material or use it for any profit-making activity or commercial gain

- You may freely distribute the URL identifying the publication in the public portal 


\title{
Modulational instability and solitons in nonlocal media with competing nonlinearities
}

\author{
B.K. Esbensen ${ }^{1}$, A. Wlotzka, M. Bache ${ }^{1}$, O. Bang ${ }^{1}$, W. Krolikowski ${ }^{2}$ \\ ${ }^{1}$ DTU Fotonik, Department of Photonics Engineering, Technical University of Denmark, Lyngby, Denmark \\ ${ }^{2}$ Department of Physics, University of Karlsruhe, Karlsruhe, Germany \\ ${ }^{3}$ Laser Physics Centre, Australian National University, Canberra, Australian Capital Territory 0200, Australia \\ wzk111@gmail.com
}

\begin{abstract}
We investigate theoretically propagation and spatial localization of light in nonlocal media with competing nonlinearities. We show that the competing focusing and defocusing nonlinearities enable coexistence of dark or bright spatial solitons in the same medium by varying the intensity of the beam.
\end{abstract}

(C) 2012 Optical Society of America

OCIS codes: $190.4420,190.6135$.

Recently there has been growing interest in the nonlocal nonlinear media where the nonlinear response of the medium in a particular location depends on the light intensity in a certain neighborhood of this location [1,2]. Nonlocal nonlinearities have been identified in a variety of physical systems where the nonlocal response is caused by either transport processes such as heat or ballistic atomic transport or nematic liquid crystals and Bose Einstein condensates with long range interaction. The concept of nonlocal nonlinearity has been recently extended to media with the socalled synthetic nonlinearities where the nonlinear response is a result of two or more competing processes or effects. Such competing nonlinear response occurs naturally in Bose Einstein condensates with dipolar interaction. It has been shown, e.g. that simultaneous presence of nonlocal nonlinearities of opposite sign leads to stabilization of complex soliton structures which are otherwise unstable in medium with one type of nonlocal nonlinearity [3]. On the other hand, it turns out that competing nonlinearities may also destabilize dark soliton states and lead to repulsion of in-phase bright solitons [4].

In this work we study analytically and numerically the modulational instability of plane waves and soliton formation in nonlocal media with competing nonlinearities. In particular, we will explore the interplay between nonlocality and the nonlinearity and its effect on stability of bright and dark solitons. We will also discuss the regime in which the nonlocal medium simultaneously support bright and dark solitons.

We will consider propagation of optical beam with the slowly varying amplitude $u(x, z)$ and corresponding intensity $I=|u(x, z)|^{2}$ in nonlinear media with the intensity-dependent refractive index change $\Delta n(x, I)=\alpha_{1} \int R_{1}(x-$ $\left.x^{\prime}\right) I\left(x^{\prime}, z\right) \mathrm{d} x^{\prime}+\alpha_{2} \int R_{2}\left(x-x^{\prime}\right) I\left(x^{\prime}, z\right) \mathrm{d} x^{\prime}$, where $x$ and $z$ represent transverse and longitudinal coordinates, respectively. $\alpha_{1}$ and $\alpha_{2}$, represent the strength and sign of the two nonlinear contributions, respectively. Here $\alpha_{1}$ will be considered positive, corresponding to a self-focusing nonlinearity, while $\alpha_{2}$ negative, corresponding to a self-defocusing nonlinearity. The nonlocal response functions $R_{1,2}(x)$ define the nonlocal character of the nonlinearity. Without loss of generality we will use Gaussian nonlocal response $R_{i}(x)=1 / \sqrt{\pi \sigma_{i}} \exp \left(-|x|^{2} / \sigma_{i}^{2}\right)$. Then the evolution of the 1dimensional beams in a phenomenological model of a nonlinear medium is represented by the following nonlocal nonlinear Schrödinger equation

$$
\mathrm{i} \partial u_{z}+\partial^{2} u_{x x}+\Delta n u=0 .
$$

Modulational instability (MI) is one of the most fundamental effects associated with wave propagation in nonlinear media. It signifies the exponential growth of a small perturbation of the plane wave (PW) amplitude during propagation. Following the standard procedure we find that the perturbation evolves with propagation as $a(x, z) \propto \exp (\lambda z)$ where the growth rate $\lambda$ is determined by the following relation

$$
\lambda^{2}=-k^{2} A_{0}^{2}\left(k^{2} / A_{0}^{2}-2 \tilde{R}(k)\right) .
$$

where $\tilde{R}(k)=\tilde{R_{1}}(k)+\tilde{R_{2}}(k)$ is the Fourier transform of the nonlocal response function. Therefore plane wave solutions are stable if perturbations at all wave numbers $k$ do not grow with propagation. This is the case as long as $\lambda$ is 

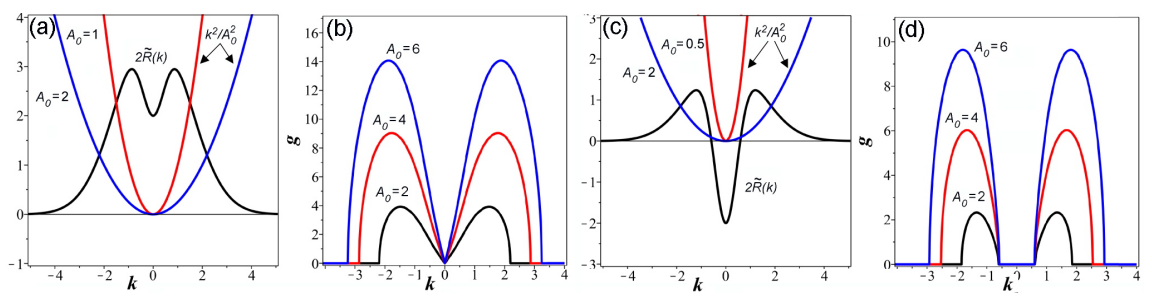

Fig. 1. (a) Plot of the functions $2 \tilde{R}(k)$ and $k^{2} / A_{0}^{2}$ for $A_{0}=1$ and $A_{0}=2$. (b) MI gain curves for $A_{0}=2, A_{0}=4$ and $A_{0}=6 . \alpha_{1}=2, \alpha_{2}=-1, \sigma_{1}=1$ and $\sigma_{2}=3$. (c) Plot of the functions $2 \tilde{R}(k)$ and $k^{2} / A_{0}^{2}$ when $\left|\alpha_{2}\right|>\alpha_{1}$, for $A_{0}=0.5$ and $A_{0}=2$; (d) The MI gain curves for $A_{0}=2, A_{0}=4$ and $A_{0}=6$. In (c-d) $\alpha_{1}=1, \alpha_{2}=-2, \sigma_{1}=1$ and $\sigma_{2}=3$.

purely imaginary. Physically, modulational stability means that small-amplitude waves can propagate along with the background intense PW, although their propagation parameter $\lambda$ depends on the PW intensity $A_{0}^{2}$. In terms of MI, the regime $\left|\alpha_{2}\right|>\alpha_{1}$ is particularly interesting, because the stability properties become intensity-dependent. If $\sigma_{2}>\sigma_{1}$ low intensity PWs are modulationally stable, whereas high intensity PWs are unstable. So we may have MI even though the strength of the defocusing nonlinearity exceeds that of the focusing, $\left|\alpha_{2}\right|>\alpha_{1}$. These unique stability properties are a pure consequence of the competition between nonlocal nonlinearities and has not been observed before. In media with just one self-defocusing (self-focusing) nonlocal nonlinearity, PWs would be stable (unstable), independently of the intensity. Since each of the individual response functions, $R_{j}$, is normalized, $\tilde{R}(0)=\alpha_{1}+\alpha_{2}$. If $\alpha_{1}>\left|\alpha_{2}\right|$ we will therefore always have $2 \tilde{R}(k)>k^{2} / A_{0}^{2}$, and thus $\lambda^{2}>0$, in a certain band symmetrically centered about the origin, for $k$ sufficiently small. So independent of the details in the behavior of the symmetric response function, we always have long wave MI in this regime [Fig.1(a,b)]. When $\left|\alpha_{2}\right|>\alpha_{1}$, the stability properties depend on the exact behavior of the response function. If $\tilde{R}(k) \leq 0$ for all $k \in \mathbb{R}$, PW solutions are modulationally stable. On the other hand, if $\tilde{R}(k)>0$ for $k \in I \subset \mathbb{R}$, PWs will be unstable if the intensity $A_{0}^{2}$ is sufficiently high [see Fig.1(c,d)].
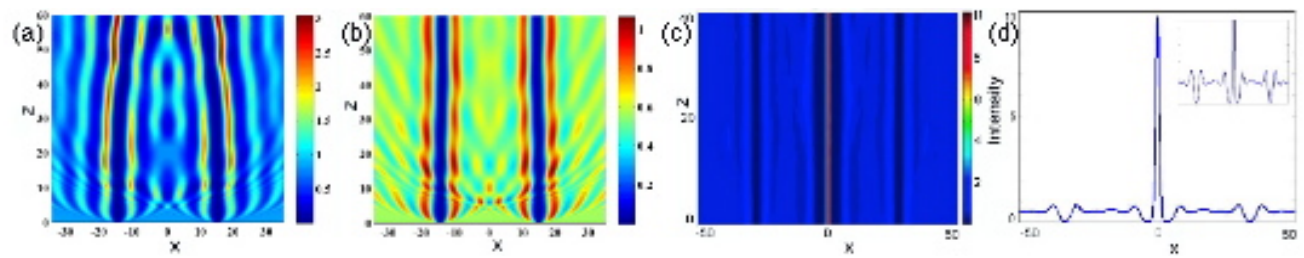

Fig. 2. (a,b) Evolution of the "dark beams" from the initial amplitude distribution $u(x, z)=A \tanh (x-$ $\left.x_{0}\right) \tanh \left(x+x_{0}\right)$. (a) Unstable background $\left(A_{0}=0.85\right)$, breakup of the initial structure and formation of bright solitons. (b) Stable background $\left(A_{0}=0.75\right)$ and formation of two dark solitons. (c-d) Coexistence of bright and dark solitons for $\alpha_{1}=1, \alpha_{2}=-2, \sigma_{1}=1$ and $\sigma_{2}=3$. (c) dynamics of soliton propagation (d) stable intensity profile. The inset depicts details of dark solitons.

As the stability properties of the plane wave depends crucially on the intensity one can envisage a situation when both bright and dark solitons may coexist on the same background as long as its intensity is below the MI threshold. Indeed such situation is illustrated in Fig.2(d) where we show the evolution of two dark and one bright solitons.

\section{References}

1. A. Snyder and J. Mitchell, Science 276, 1538 (1997).

2. W. Krolikowski, O. Bang, N. I. Nikolov, D. Neshev, J. Wyller, J. J. Rasmussen, and D. Edmundson, J. Opt. B: Quantum Semiclass. Opt. 6, S288-S294 (2004).

3. D. Mihalache, D. Mazilu, F. Lederer, L.C. Crasovan, Y.V. Kartashov, L. Torner, B.A. Malomed, Phys. Rev. E 74, 066614 (2006); J. Cuevas, B.A. Malomed, P.G. Kevrekidis, D.J. Frantzeskakis, Phys. Rev. A 79, 053608 (2009).

4. Y. Du, Z. Zhou, H. Tian and D. Liu, J. Opt. 13, 015201 (2010). 